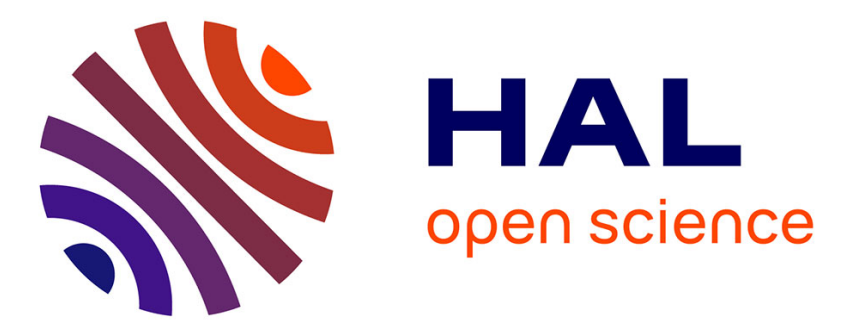

\title{
Risk Factors for Idiopathic Orbital Inflammation: A Case-control Study
}

Ward Rogier Bijlsma, Carla van Gils, Dion Paridaens, Maarten P Mourits, Rachel Kalmann

\section{- To cite this version:}

Ward Rogier Bijlsma, Carla van Gils, Dion Paridaens, Maarten P Mourits, Rachel Kalmann. Risk Factors for Idiopathic Orbital Inflammation: A Case-control Study. British Journal of Ophthalmology, 2010, 95 (3), pp.360. 10.1136/bjo.2009.175190 . hal-00567047

\section{HAL Id: hal-00567047 https://hal.science/hal-00567047}

Submitted on 18 Feb 2011

HAL is a multi-disciplinary open access archive for the deposit and dissemination of scientific research documents, whether they are published or not. The documents may come from teaching and research institutions in France or abroad, or from public or private research centers.
L'archive ouverte pluridisciplinaire HAL, est destinée au dépôt et à la diffusion de documents scientifiques de niveau recherche, publiés ou non, émanant des établissements d'enseignement et de recherche français ou étrangers, des laboratoires publics ou privés. 


\section{Risk Factors for Idiopathic Orbital Inflammation: A Case-control Study}

Ward R. Bijlsma, MD; Carla H. van Gils, PhD; Dion Paridaens, MD, PhD; Maarten P. Mourits, MD, PhD; Rachel Kalmann, MD, PhD

Author Affliations: Department of Ophthalmology (Drs Bijlsma and Dr Kalmann) and Julius Center for Health Sciences and Primary Care (Dr van Gils), University Medical Center Utrecht, Utrecht, the Netherlands; Rotterdam Eye Hospital, Rotterdam, the Netherlands (Dr

Paridaens); and Department of Ophthalmology, Academic Medical Center, Amsterdam, the Netherlands (Prof Dr Mourits).

Correspondence: Ward R. Bijlsma, MD, Department of Ophthalmology, University Medical Center Utrecht, Heidelbergiaan 100, 3584 CX Utrecht, the Netherlands (w.r.bijlsma@umcutrecht.nl).

Keywords: idiopathic orbital inflammation, orbital pseudotumor, risk factors, case-control studies

Word count: ca 2550. 


\section{[Abstract]}

Objective: To identify risk factors involved in the development of idiopathic orbital inflammation (IOI).

Methods: Case-control study of 69 adults who had had a first episode of IOI and 296 adult controls with rhegmatogenous retinal detachment (RD) selected from 3 orbital centers in the Netherlands between 2000 and 2006. Participants filled out a questionnaire on demographic factors, medical history, health status, and exposures for the 2 years prior to disease presentation. In addition, women were questioned about previous or current pregnancies and their hormonal status. Odds ratios (ORs) and accompanying 95\% confidence intervals (CIs) for IOI in relation to potential risk factors such as body mass index (BMI), bisphosphonates, and autoimmune disease were estimated. ORs were adjusted for age, sex, socioeconomic status, smoking, and blunt orbital trauma using logistic regression. Analyses were carried out both with and without multiple imputation of missing values.

Results: The risk of IOI was increased in participants who had a higher BMI (third vs first tertile: OR, 2.88; 95\% CI, 1.32-6.32) and in participants who used bisphophonates (OR, 8.68; 95\% CI, 1.16-65.0). The risk was decreased in participants with a higher socioeconomic status (third vs first tertile: OR, 0.38; 95\% CI, 0.17-0.84) and in women who were older at first childbirth (third vs first tertile: OR, 0.14; 95\% CI, 0.03-0.64). A close to significant association was found for IOI and autoimmune disease (OR, 2.56; 95\% CI, 0.93-7.05). 
Conclusions: IOI is associated with lower socioeconomic status, higher BMI, and use of oral bisphosphonates. In women, IOI is also associated with younger age at first childbirth. 


\section{[Introduction]}

Idiopathic orbital inflammation (IOI) is among the most frequent orbital diseases encountered by ophthalmologists. ${ }^{1}$ IOI is a noninfectious inflammation of the orbital soft tissues for which no cause is found after local and systemic evaluation. ${ }^{2}$ The term IOI refers to a collection of different entities, including idiopathic sclerosing orbital inflammation, ${ }^{3}$ idiopathic granulomatous orbital inflammation, ${ }^{4}$ dacryoadenitis, ${ }^{5}$ and orbital myositis, ${ }^{6}$ which makes patients with IOI an inhomogeneous patient population.

IOI presents with various signs and symptoms of inflammation, most frequently pain, eyeball motility disturbances, and proptosis. ${ }^{2}$ Due to the orbital soft tissue swelling, IOI may mimic a neoplasm ("orbital pseudotumor" is the historical term for IOI). The clinical course of IOI ranges from mild and self-limiting to devastating orbital sclerosis with blindness. ${ }^{2}$

IOI, as is indicated by the word "idiopathic" in the disease name, is of unknown etiology. Many case reports and series have highlighted possible etiologic factors for IOI, including autoimmune diseases ${ }^{7}$ and medications such as bisphosphonates, ${ }^{8}$ lithium, ${ }^{9}$ and chemotherapeutics. ${ }^{10}$ Retroperitoneal fibrosis is a systemic disease entity that is similar in many respects to IOI. ${ }^{11}$ Ergot derivatives, ${ }^{12,13}$ asbestos, ${ }^{14}$ and a genetic predisposition ${ }^{15}$ have been reported as risk factors for retroperitoneal fibrosis.

To our knowledge, systematic research of the risk factors for IOI has not been previously conducted. In this case-control study, we explore associations between a number of risk factors and IOI.

Risk factors idiopathic orbital inflammation, version 200910 


\section{METHODS}

This study was designed as a case-control study.

Our patients came from 3 orbital clinics in the Netherlands between 2000 and 2006. Patients with IOI were identified by searching the hospital diagnosis database for ICD-9 code 376.1 . ICD-9 code 376.0, which was primarily used for orbital infection, was excluded.

Patient records were reviewed and patients with all of the following 3 criteria were included in the study: (1) a clinical picture of orbital inflammation with either no improvement after antibiotic therapy and prompt improvement after systemic prednisone, or nonspecific inflammation after an orbital tissue biopsy; (2) no local or systemic identifiable cause of the inflammation; and (3) age 18 or older and residing in the Netherlands. For example, Sarcoidosis, lupus, and Wegener granulomatosis were excluded as causes of IOI. Only patients who had had a first episode of IOI were included in the study. The localisation of IOI was determined by reviewing radiology images and was categorized as localized myositis, localized dacryoadenitis, or diffuse inflammation. Histology reports, when available, were reviewed and classified as classic, sclerosing, or granulomatous inflammation ${ }^{7}$. Laterality andcorticosteroid treatment were recorded.

For the controls, adults who had had a first episode of a rhegmatogenous retinal detachment (RD) were identified using the hospital surgical database. For each patient with IOI, we randomly selected 4 controls who had been diagnosed with RD in the same hospital and in the same year and month as the patient. Hospital-based controls were chosen for availability, similar geographic area, high response rate, and similar recollection of information. These 
controls were considered to be a good representation of the study base. Moreover, rhegmatogenous RD was considered to be of mechanical etiology and expected to share none or few etiologic factors with IOI.

Institutional review board approval was given for this study, which adhered to the tenets of the Declaration of Helsinki.

A 6-page questionnaire was sent to all patients and controls. The questionnaire collected information on sex, age at diagnosis, body mass index (BMI) at diagnosis, smoking status (current, former, never), pack years of smoking, diabetes (yes/no), and average number of days with influenza or colds per year. Socioeconomic status was determined by the highest educational level of the participant (primary school, secondary school, professional education, higher education), and, when the information was available, by the highest educational level of the participant's partner. To determine whether an autoimmune disease was or had been present, participants were asked to describe prior inflammation of the orbit, muscles, joints, skin, connective tissue, neural tissue, intestines, kidneys, lungs, and/or thyroid up to the time of the questionnaire. An immunologist determined whether the self-reported inflammation was consistent with autoimmune disease.

The following questions were asked about the 2 years preceding the diagnosis of RD or IOI, all defined as yes/no variables: any occurrence of blunt trauma to the orbit; any surgery of the orbit, sinuses, or facial bones; any use of bisphosphonates, ergot derivatives, lithium, chemotherapy, or thyroid hormones; any exposure to asbestos or lysergic acid diethylamide (LSD); or any reported physical or emotional distress. In addition, participants were asked if 
any siblings, parents, or grandparents had ever had any orbital inflammatory disease, lupus, thyroiditis, myasthenia gravis, uveitis, or diabetes mellitus. Women were asked about the number of pregnancies, age at birth of their first child, use of oral contraceptives or hormone replacement therapy during the 2 years preceding diagnosis, menopausal status at disease development, and age at menopause, if applicable. A time period of 2 years before the onset of the disease was chosen for adequate recall and to look at relative short-term effects of potential etiologic factors.

One reminder was sent to participants who did not respond to the first request. The response forms were checked for sex and age to identify forms that were not filled out by the intended individual. Nonmatching forms were excluded.

Response forms were entered into a database and analyzed using statistical software (SPSS for Windows 15.0 and R 2.6.0 for Windows). Characteristics of responders and nonresponders were compared. Continuous variables were categorized into tertiles. Using complete case analysis, odds ratios (ORs) and accompanying 95\% confidence intervals (CIs) were computed to describe the associations between risk factors and the occurrence of IOI. Multivariate analysis was performed using binary logistic regression to adjust relationships for age (continuous), sex, tertiles of socioeconomic status, pack-year tertiles of smoking, and blunt orbital trauma because adjustment for these variables changed the ORs more than $10 \%$.

Multivariate analysis was repeated on a multiple imputed dataset to obtain more precise and valid measures of association for variables with missing values. ${ }^{16}$ Multiple imputation was chosen because of its wide applicability to almost any statistical situation. ${ }^{17}$ The pattern of 
missing values was evaluated and considered missing at random. For each missing value, 10 imputations were performed using between 6 and 19 best-correlated variables, including the outcome. ${ }^{18}$ Imputation was carried out in R (with the aregImpute function in library "Hmisc").

\section{RESULTS}

Questionnaires were sent by mail to 103 patients and 410 controls. Two questionnaires to controls under the age of 18 were not sent because of their age. Sixty-nine patients (67\%) and 295 controls (72\%) returned the questionnaires after 2 mailings. Of the nonresponders, 16 (all controls) had died and 16 (4 patients; 12 controls) had moved without providing a new address. Patients who responded were somewhat older than patients who did not respond (average age, 52.6 years vs 49.0 years) (Table 1). Response rates of patients and controls differed somewhat between clinics (patients from clinic B responded less) and between years of diagnosis (controls diagnosed between 2002 and 2003 responded less).

The patients with IOI were categorized as follows: 17 with isolated myositis, 19 with isolated dacryoadenitis, and 33 with diffuse inflammation. Biopsies showed classic inflammation in 21 , sclerosing in 8 , and granulomatous in 2 patients. Five patients had bilateral orbital involvement. Fifty-one patients were treated with corticosteroids.

The mean (SD) age at diagnosis of the patients with IOI was 52.6 (13.4) years. For controls with $\mathrm{RD}$, the mean (SD) age was $59.6(11.8)$ years. The male to female ratio was 2:3 in patients with IOI and 7:4 in controls with RD.

Potential risk factors for IOI are described for patients and for controls. Patients with IOI were younger, more often female, and of lower socioeconomic status. In the multivariate 
analysis, we adjusted the effect of other potential risk factors for the confounding effect of age (continuous variable), sex, and socioeconomic status (tertiles). In addition, we adjusted for pack years (tertiles) of smoking and for blunt trauma to the orbit.

The risk of IOI was lower for those with higher socioeconomic status (third vs first tertile: adjusted OR, 0.36; 95\% CI, 0.16-0.81). The risk increased with higher BMI (third vs first tertile: adjusted OR, 2.88; 95\% CI, 1.32-6.32). Use of bisphosphonates was associated with IOI (adjusted OR, 8.68; 95\% CI, 1.16-65.0) with imprecision due to low absolute numbers of bisphosphonate users. The association between bisphosphonate use and IOI persisted in the stratum of postmenopausal females (adjusted OR, 9.29; 95\% CI, 1.98-72.0), after additional adjustment for female hormone supplements (adjusted OR, 9.35; 95\% CI, 1.21-72.5). A trend for association between IOI and autoimmune disease was found, although it was not statistically significant (adjusted OR, 2.56; 95\% CI, 0.93-7.05).

Female patients were more often premenopausal at diagnosis than female controls, but this association disappeared after adjustment for age, socioeconomic status, smoking, and trauma (adjusted OR, 1.07; 95\% CI, 0.22-5.19). For women who had given birth, a higher age at first childbirth was associated with a lower risk of IOI (third vs first tertile: adjusted OR, 0.15; 95\% CI, 0.03-0.72). The other variables in Table 1 did not show a clear relationship with IOI. Little seasonal variation of IOI presentation was found with $26 \%$ presenting in winter, $22 \%$ in spring, $25 \%$ in summer, and $28 \%$ in autumn.

Multiple imputation changed the association measures on average by $16.5 \%$ to a weaker association in 23 variables and a stronger association in 12 variables. 


\section{COMMENT}

In this study, risk factors for the development of IOI are evaluated by comparing patients with IOI to controls with RD. We found a significant association between IOI and sex (female), age (younger), socioeconomic status (lower), BMI (higher), use of bisphosphonates, and age at birth of first child (younger). The association between autoimmune disease and IOI was close to statistically significant.

In the IOI group there were relatively more females (61\%) than in the RD group (39\%), and the patients with IOI were, on average, 7 years younger than the controls with RD (mean age, 53 and 60 years, respectively). These differences highlight the different demographic features of IOI and RD, where RD is associated with male sex and older age. ${ }^{19}$

We used the highest level of education of the patient and of his or her partner as a surrogate variable for socioeconomic status. RD has been associated with a lower education level (education beyond age 16 years: OR, 0.6; 95\% CI, 0.3-1.1). ${ }^{19}$ This association may be explained by ocular trauma as a cause of $\mathrm{RD}\left(10 \%\right.$ in a large survey of $\mathrm{RD}^{20}$; it was $6 \%$ in our study), and a higher risk of ocular trauma in craftsmen. However, in our study, we found lower educational levels to be associated with IOI as well, even after adjusting for ocular trauma.

As is known from clinical practice, a higher BMI was associated with IOI. The BMI was calculated from height and weight at diagnosis of IOI and, therefore, should not have been affected by the use of corticosteroids. The relation between metabolic regulation and the immune system has been the interest of recent research. Obesity is associated with a chronic 
inflammatory response. In obesity, the inflammatory response appears to be triggered and reside predominantly in adipose tissue. The high orbital fat content in obese patients may explain why inflammatory diseases occur in the orbit. ${ }^{21}$ Leptin is thought to be central to the link between obesity and autoimmunity because leptin is secreted by adipocytes and can trigger the production of proinflammatory and pathogenic cytokines. ${ }^{22}$

The cases of 3 patients who developed IOI after administration of intravenous bisphosphonates have been reported. ${ }^{8,23}$ The proposed mechanism of action is the release of the inflammatory cytokines IL-1 and IL-6 triggered by bisphosphonates. The prevalence of bisphosphonate use in the population is low (it was $2.8 \%$ in our study). Therefore, the association between bisphophonate use and IOI will not have major clinical consequences. We decided to look at the role of female hormones in IOI because of the intimate relationship between hormones and the immune system. Estrogens are implicated as enhancers of humoral immunity, and androgens and progesterone are natural immune suppressors. ${ }^{24 ; 25} \mathrm{We}$ looked for higher estrogen levels in patients with IOI, which is suggested by a small female predilection and higher age at menopause. ${ }^{24}$ However, the observation of a lower number of pregnancies in patients with IOI, when estrogens are high, does not support an etiologic role of high estrogens in IOI. The statistically significant association between the lower age at first childbirth and IOI suggests that female hormones play a role in IOI, but it is not clear how this relates to estrogens and progesterone. Lower age at first childbirth may also be a risk indicator of risk factor clustering, such as occurs with breast cancer. ${ }^{26}$

An association between IOI and autoimmune diseases was postulated by Mombaerts and Koornneef, ${ }^{27}$ who found that $10 \%$ of patients with IOI had a concurrent autoimmune disease. We found concurrent autoimmune diseases in $12 \%$ of patients with IOI (OR, 2.56; 95\% CI, 
0.93-7.05). Although the association was not statistically significant, it is suggestive of an autoimmune pathogenesis in IOIA genetic predisposition or a disregulated immune system with autoantibodies against multiple self-antigens could explain the high co-occurrence of IOI with autoimmune diseases.

Of the proposed possible risk factors, we did not find an association between IOI and ergot derivatives, lithium, LSD, chemotherapy, asbestos, trauma, or family history. The equal number of days patients with IOI and controls with RD had influenza or colds suggests that they had similar immune system functioning.

A case-control study is implicitly limited in that only associations can be described, but no causality inferred. The quality of such a study is highly dependent on the selection of controls. In this study, it is not possible to draw conclusions about common risk factors between IOI and RD. Because RD is caused by posterior vitreous detachment, we thought RD unlikely to be associated with risk factors other than age, sex, and ocular trauma. We did not use a control group of healthy volunteers because we expected an unacceptable low response rate. A cohort design was considered inefficient because of the long inclusion time of 7 years.

By selecting all patients with IOI (some who were not histologically confirmed), we have selected a heterogeneous group of diseases. This will likely dilute associations of risk factors that are specific for subgroups like dacryoadenitis and myositis. However, the sample size is too low for subgroup analysis. Limiting the study to histologically confirmed patients would have introduced a selection bias and would have yielded a lower number of cases. 
Recall bias due to the retrospective nature of this study was considered to have influenced both patients and controls in equal amounts. The effect of missing values was evaluated by using multiple imputation. Multiple imputations caused the associations to attenuate in a majority of variables. A group of variables that was largely affected by multiple imputations were variables measured only on females, which is probably due to the lower number of observations. Multiple imputations did not alter the conclusions but might have resulted in more valid and precise effect estimates. ${ }^{16}$

In conclusion, this study describes the first systematic search for risk factors for IOI. Novel and statistically significant associations with IOI are found between lower socioeconomic status, higher BMI, use of oral bisphosphonates, and a lower age at first childbirth in women. An important and close to statistically significant association has been found between autoimmune diseases and IOI. 
Financial Disclosure/Funding/Support: This research was facilitated by a grant from the F.P. Fischer Foundation.

Copyright licence statement: The Corresponding Author has the right to grant on behalf of all authors and does grant on behalf of all authors, an exclusive licence (or non exclusive for government employees) on a worldwide basis to the BMJ Publishing Group Ltd to permit this article (if accepted) to be published in BJO and any other BMJPGL products and sublicences such use and exploit all subsidiary rights, as set out in our licence (http://group.bmj.com/products/journals/instructions-for-authors/licence-forms).

Competing interest: None declared 
Table 1. Characteristics of Responders and Nonresponders Among Patients and Controls

\section{Patients}

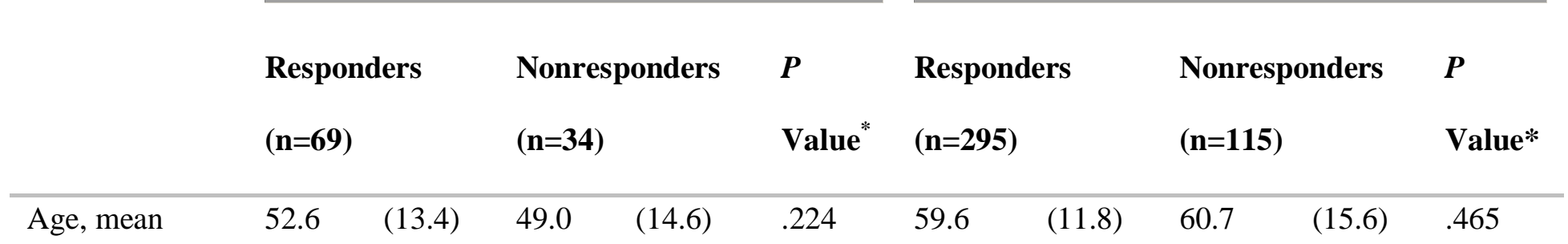

(SD), y

Male sex

29

Orbital clinic

$\begin{array}{lllll}\text { A } & 12 & (17 \%) & 8 & (24 \%) \\ \text { B } & 24 & (35 \%) & 17 & (50 \%) \\ \text { C } & 33 & (48 \%) & 9 & (26 \%)\end{array}$

Years of

.676

\section{Controls}

$\begin{array}{ccccc}.832 & 190 & (64 \%) & 73 & (64 \%) \\ .116 & & & & \\ & 53 & (18 \%) & 26 & (23 \%) \\ 125 & (42 \%) & 38 & (33 \%) \\ & 117 & (40 \%) & 51 & (44 \%)\end{array}$

diagnosis 


$\begin{array}{lllllllll}2000-2001 & 8 & (12 \%) & 3 & (9 \%) & 30 & (10 \%) & 14 & (12 \%) \\ 2002-2003 & 16 & (23 \%) & 7 & (21 \%) & 54 & (18 \%) & 38 & (33 \%) \\ 2004-2005 & 23 & (33 \%) & 9 & (26 \%) & 102 & (35 \%) & 26 & (23 \%) \\ 2006-2007 & 22 & (32 \%) & 15 & (44 \%) & 109 & (37 \%) & 37 & (32 \%)\end{array}$

$* P$ values calculated by $\chi^{2}$ test.

SD standard deviation, y year 


\section{References}

1. Weber AL, Romo LV, Sabates NR. Pseudotumor of the orbit. Clinical, pathologic, and radiologic evaluation. Radiol.Clin.North Am. 1999;37:151-68, xi.

2. Yuen SJ, Rubin PA. Idiopathic orbital inflammation: distribution, clinical features, and treatment outcome. Arch.Ophthalmol. 2003;121:491-9.

3. Hsuan JD, Selva D, McNab AA et al. Idiopathic sclerosing orbital inflammation. Arch.Ophthalmol. 2006;124:1244-50.

4. Mombaerts I, Schlingemann RO, Goldschmeding R et al. Idiopathic granulomatous orbital inflammation. Ophthalmology 1996;103:2135-41.

5. Mombaerts I, Schlingemann RO, Goldschmeding R et al. The surgical management of lacrimal gland pseudotumors. Ophthalmology 1996;103:1619-27.

6. Mannor GE, Rose GE, Moseley IF et al. Outcome of orbital myositis. Clinical features associated with recurrence. Ophthalmology 1997;104:409-13.

7. Mombaerts I, Goldschmeding R, Schlingemann RO et al. What is orbital pseudotumor? Surv.Ophthalmol. 1996;41:66-78.

8. Subramanian PS, Kerrison JB, Calvert PC et al. Orbital inflammatory disease after pamidronate treatment for metastatic prostate cancer. Arch.Ophthalmol. 2003;121:1335-6.

9. Dick AD, Atta H, Forrester JV. Lithium-induced orbitopathy. Arch.Ophthalmol. 1992;110:4523. 
10. Fortin D, Salame JA, Desjardins A et al. Technical modification in the intracarotid chemotherapy and osmotic blood-brain barrier disruption procedure to prevent the relapse of carboplatin-induced orbital pseudotumor. AJNR Am.J.Neuroradiol. 2004;25:830-4.

11. McCarthy JM, White VA, Harris G et al. Idiopathic sclerosing inflammation of the orbit: immunohistologic analysis and comparison with retroperitoneal fibrosis. Mod.Pathol. $1993 ; 6: 581-7$

12. Mitchinson MJ. Methysergide and retroperitoneal fibrosis. Lancet 1987;1:870.

13. Shaunak S, Wilkins A, Pilling JB et al. Pericardial, retroperitoneal, and pleural fibrosis induced by pergolide. J.Neurol.Neurosurg.Psychiatry 1999;66:79-81.

14. Uibu T, Oksa P, Auvinen A et al. Asbestos exposure as a risk factor for retroperitoneal fibrosis. Lancet 2004;363:1422-6.

15. Martorana D, Vaglio A, Greco P et al. Chronic periaortitis and HLA-DRB $1 * 03$ : another clue to an autoimmune origin. Arthritis Rheum. 2006;55:126-30.

16. Donders AR, van der Heijden GJ, Stijnen T et al. Review: a gentle introduction to imputation of missing values. J.Clin.Epidemiol. 2006;59:1087-91.

17. Allison PD. Missing data techniques for structural equation modeling. J.Abnorm.Psychol. 2003;112:545-57.

18. Arnold AM, Kronmal RA. Multiple imputation of baseline data in the cardiovascular health study. Am.J.Epidemiol. 2003;157:74-84.

19. Austin KL, Palmer JR, Seddon JM et al. Case-control study of idiopathic retinal detachment. Int.J.Epidemiol. 1990;19:1045-50. 
20. Haimann MH, Burton TC, Brown CK. Epidemiology of retinal detachment. Arch.Ophthalmol. 1982;100:289-92.

21. Bouloumie A, Curat CA, Sengenes $\mathrm{C}$ et al. Role of macrophage tissue infiltration in metabolic diseases. Curr.Opin.Clin.Nutr.Metab Care 2005;8:347-54.

22. Matarese G, Procaccini C, De R, V. The intricate interface between immune and metabolic regulation: a role for leptin in the pathogenesis of multiple sclerosis? J.Leukoc.Biol. 2008;84:893-9.

23. Ryan PJ, Sampath R. Idiopathic orbital inflammation following intravenous pamidronate. Rheumatology.(Oxford) 2001;40:956-7.

24. Cutolo M, Capellino S, Sulli A et al. Estrogens and autoimmune diseases. Ann.N.Y.Acad.Sci. 2006;1089:538-47.

25. Cutolo M, Villaggio B, Craviotto C et al. Sex hormones and rheumatoid arthritis. Autoimmun.Rev. 2002;1:284-9.

26. Soerjomataram I, Pukkala E, Brenner $\mathrm{H}$ et al. On the avoidability of breast cancer in industrialized societies: older mean age at first birth as an indicator of excess breast cancer risk. Breast Cancer Res.Treat. 2008;111:297-302.

27. Mombaerts I, Koornneef L. Current status in the treatment of orbital myositis. Ophthalmology 1997;104:402-8. 